\title{
A survey of phenotype distribution of subgroup A2, blood groups MNS, P, and Kell in the Kazak nationality living in northern Xinjiang of China
}

\author{
Fen Qiu ${ }^{1}$, Gulibahati ${ }^{1}$, Jun Wen ${ }^{2 *}$, Xuemei $\operatorname{Tian}^{3}$, Asmuguli ${ }^{1}$, Hui Pan ${ }^{1}$ \\ ${ }^{1}$ Department of Transfusion, Kuitun Hospital of Xinjiang's Yili Kazak Autonomous Prefecture, Kuitun, Xinjiang, 833200, China; \\ ${ }^{2}$ Department of Transfusion, Xinjiang Uygur Autonomous Region People's Hospital, Urumqi, Xinjiang, 830000, China; \\ ${ }^{3}$ Department of Transfusion, Friendship Hospital of Xinjiang's Yili Kazak Autonomous Prefecture, Yili, Xinjiang, 835000, China.
}

\begin{abstract}
The research was undertaken to study the phenotypic polymorphisms of the subgroup A2, blood groups MNS, $\mathrm{P}$, and Kell in the Kazakh population in northern Xinjiang, China and establish data on rare blood group antigens in the Kazakh population, in order to provide references for clinical blood transfusion safety and prevention of hemolytic disease of the new born. In this study, 6,862 unrelated Kazakh individuals in northern Xinjiang were randomly selected, and their blood samples were collected for serological testing. The antigens of $\mathrm{A}, \mathrm{B}, \mathrm{Al}, \mathrm{M}$, $\mathrm{N}, \mathrm{Pl}$ and $\mathrm{K}$ were detected by serological saline tube method, and the antigens of $\mathrm{S}$, s, and $\mathrm{k}$ were detected by the microcolumn gel antiglobulin card method. The results were as follows: (1) The detection rates of subgroup A2 in group A and group AB were $7.08 \%$ and $21.79 \%$, respectively; (2) The allele frequencies of the blood groups MNS, $\mathrm{P}$ and Kell were $\mathrm{M}=0.5668, \mathrm{~N}=0.4332, \mathrm{~S}=0.1860, \mathrm{~s}=0.8140, \mathrm{P} 1=0.2848, \mathrm{P} 2=0.7152, \mathrm{~K} 1=0.0096, \mathrm{~K} 2=0.9904$. The observed values and expected values of frequency distribution of genotypes were compared by $\chi^{2}$ test, which conformed to the Hardy-Weinberg genetic law $(P>0.05)$; (3) Fourteen cases of $\mathrm{S}^{-} \mathrm{S}^{-}$rare phenotype were detected in MNS blood group system, with a frequency of $1.16 \%$; (4) The frequency of $\mathrm{K}$ antigen in the Kell blood group system was $1.92 \%$. One case of rare KK homozygote was detected, with a frequency of $0.034 \%$. Our study suggested that the distribution of gene frequency of subgroup A2, blood groups MNS, P and Kell in the Kazakh population in northern Xinjiang has its own characteristics, and their blood group MNS has unique genotypes. The positive rate of $\mathrm{K}$ antigen of blood group Kell in the Kazakh population was significantly higher than Chinese Han population.
\end{abstract}

Keywords: RBC blood group, subgroup A2, blood groups MNS, P, Kell, gene frequency

\section{INTRODUCTION}

Blood group is one of the most important genetic markers. Xinjiang is a multi-ethnic living area: of which Kazakh is the second largest minority, whose population is mainly distributed in the northern Xinji-

*Correspondence to: Jun Wen, Department of Transfusion, Xinjiang Uygur Autonomous Region People’s Hospital, Urumqi 830000, China. Email:418216181@qq.com.

Conflict of interests: The authors have declared no conflict of interests. ang. This population accounts for $94.27 \%$ of the total number of the Kazakhs in Xinjiang. Due to the lack of data on the distribution of major rare blood groups and genotypes in the Kazakh population, our team carried out research on the subgroup A2, MNS, P and Kell blood group systems of the Kazakh tribes in the northern Xinjiang in 2014. The purpose was to discover the relationship between their genetic composition and population distribution frequency by investigating the phenotype frequency and gene frequency. 
These results have provided references for the scientific, safe, and reasonable clinical blood transfusion. The results of the investigation in the past three years are reported as follows.

\section{MATERIALS AND METHODS}

\section{Sample sources}

A total of 6,862 healthy unrelated individuals from different Kazakh tribes in the Yili Kazakh Autonomous Prefecture (Altay region, Tacheng region, and Yili region: where the Kazakh population in northern Xinjiang is mainly distributed) were investigated from May 2014 to February 2017. The individuals were from 13 to 19 years old, including 3,515 males and 3,347 females. A $3-\mathrm{mL}$ blood sample was taken from each volunteers (from the elbow vein) and collected in EDTA- $\mathrm{K}_{2}$ tubes and stored in a refrigerator at $2 \sim 8^{\circ} \mathrm{C}$. Within one week, batch tests and records were completed.

\section{Reagents and instruments}

All the antibodies were provided by Shanghai Hemo Pharmaceutical Biological Co., Ltd., including antiA, anti-B (Lot.\# 20131122, 20140204, 20151224), anti-Al (Lot.\# 20140124, 20161219), anti-M (Lot.\# 20140103), anti-N (Lot.\# 20130424, 20160811), and IgM anti-Pl monoclonal antibodies (Lot.\# 20140401, 20161128), antiglobulin reagent $\mathrm{IgG}+\mathrm{C} 3 \mathrm{~d}$ (Lot.\# 20140424, 20141203, 20165002), IgG anti-D monoclonal antibodies (Lot.\# 20130904, 20151224). IgM anti-K antibody (Lot.\# TRJ1201G, Millipore, Britain; Lot.\# 8000189940, Sanqiun, Netherlands), IgG anti-S, anti-s, and anti-k antibodies (Lot.\# 42800, 8000172751, 8000189940, 8000197723, 20151129, DIAGAST, France, Sanqiun, Netherlands, and Libo Pharmaceutical Biotechnology Co., Ltd., Jiangyin, China). All the reagents were used within the validity period. Antiglobulin microcolumn test card (Lot.\# 20120308, Changchun Boxun Biotechnology Co., Ltd., Changchun, China), KA-2200 centrifuge (Kubota, Japan), TD-3A centrifuge and FYQ incubator (Changchun Boyan Scientific Instrument Co., Ltd., Changchun, China), HH.W 21.CU 600 electric heated water bath (Shanghai Medical Instrument Factory, Shanghai, China), CX31 microscope (Olympus, Japan).

\section{ABO blood group forward typing}

The screening and identification of subgroups A2 and $\mathrm{A} 2 \mathrm{~B}$ were carried out according to the Blood Transfusion Technical Manual ${ }^{[1]}$.

\section{Detection of A1, M, N, P1, and $\mathrm{K}$ antigens}

The subject's red blood cells(RBCs) suspension
$20 \mu \mathrm{L}$ (1\% 2\% in normal saline) and IgM antibody $20 \mu \mathrm{L}$ were added to the tubes, mixed and placed at room temperature for 15 minutes and centrifuged. The absence of agglutination was taken as a negative result, otherwise the result was deemed positive.

\section{Detection of S, s, and $k$ antigens}

The RBCs were formulated to $0.8 \% \sim 1.0 \%$ cell suspension. The card type microcolumn gel antiglobulin method was used to detect the S, s, k antigens according to the instructions. The RBCs' antigens (heterozygous) were selected from Sanqiun or spectrum cells for comparison. Suspicious specimens were tested repeatedly.

\section{Statistical analysis}

The gene frequency of blood groups MNS and Kell was analyzed by the gene counting method according to the procedure outlined in Genetics of $\mathrm{Hu}-$ man Blood Groups ${ }^{[2]}$. The gene frequency of blood group $\mathrm{P}$ was analyzed by square root method. The Hardy-Weinberg $(\mathrm{H}-\mathrm{W})$ equilibrium test was used to detect whether there was a significant difference between the expected value and the observed value of the phenotypic distribution of each blood group system: $c^{2}=(\text { observed value-expected value })^{2} /$ expected value, $P<0.05$ was considered as statistically significant.

\section{RESULTS}

\section{Distribution of subgroups A2 and A2B}

By screening 6,862 Kazakh people's ABO blood group in northern Xinjiang: 1,920 group A samples were detected, including 1,784 samples of subgroup Al (92.92\%) and 136 samples of subgroup A2 (7.08\%); 615 samples of $\mathrm{AB}$ were detected, including 481 samples of subgroup AlB (78.21\%) and 134 samples of subgroup A2B (21.79\%).

Phenotype and gene frequency distribution of blood groups MNS, $\mathbf{P}$ and Kell of the Kazakh population in Xinjiang

The distribution of the MN blood group was characterized as $\mathrm{MN}>\mathrm{M}>\mathrm{N}$. Fourteen cases of rare phenotype $\mathrm{S}^{-} \mathrm{s}^{-}$with a frequency of $0.74 \%$, and one case of phenotype KK with a frequency of $0.034 \%$ were detected. There was no significant difference between the expected value and observed value in the phenotypic distribution of the three blood group systems as found by the Hardy-Weinberg equilibrium test $(P>0.05)$ (Table 1). 
A survey of phenotype distribution of subgroup A2, blood groups MNS, $P$, and Kell in the Kazak

161

nationality living in northern Xinjiang of China, 2018, 2(3)

Table 1 MNS, $\mathbf{P}$ and Kell blood group phenotypes and gene frequencies of the Kazakhs population in northern Xin-

\begin{tabular}{|c|c|c|c|c|c|}
\hline Blood type system & Phenotype & Observed value $[n(\%)]$ & Expected value $[n(\%)]$ & $\mathrm{H}-\mathrm{W}$ test $P$ value & Gene frequency \\
\hline \multirow[t]{10}{*}{ MNS } & MM & $1,170(32.77)$ & $1,147.04(32.13)$ & 0.4596 & $\mathrm{M}=0.5668$ \\
\hline & $\mathrm{NN}$ & 693(19.41) & 669.73(18.76) & 0.8085 & $\mathrm{~N}=0.4332$ \\
\hline & MN & $1,707(47.82)$ & 1,753.23(49.11) & 1.2190 & \\
\hline & \multirow{2}{*}{ Total } & \multirow{2}{*}{$3,570(100.00)$} & \multirow{2}{*}{$3,570.00(100.00)$} & 2.4871 & \\
\hline & & & & $P>0.05$ & \\
\hline & SS & $73(3.90)$ & 64.73(3.46) & 1.0566 & $S=0.1860$ \\
\hline & Ss & $550(29.40)$ & $566.55(30.28)$ & 0.4835 & $\mathrm{~s}=0.8140$ \\
\hline & ss & $1,248(66.70)$ & $1,239.72(66.26)$ & 0.0553 & \\
\hline & \multirow{2}{*}{ Total } & \multirow{2}{*}{$1,871(100.00)$} & \multirow{2}{*}{ 1,871.00(100.00) } & 1.5954 & \\
\hline & & & & $P>0.05$ & \\
\hline \multirow[t]{3}{*}{$\mathrm{P}$} & Pl & $1,016(48.85)$ & $1,016(48.85)$ & NA & $\mathrm{Pl}=0.2848$ \\
\hline & P2 & 1,064(51.15) & $1,064(51.15)$ & NA & $\mathrm{P} 2=0.7152$ \\
\hline & Total & $2,080(100.00)$ & $2,080.00(100.00)$ & & \\
\hline \multirow[t]{5}{*}{ Kell } & KK & $1(0.034)$ & $0.27(0.009)$ & 1.9737 & $K=0.0096$ \\
\hline & $\mathrm{Kk}$ & $55(1.889)$ & $55.37(1.902)$ & 0.0025 & $\mathrm{k}=0.9904$ \\
\hline & $\mathrm{kk}$ & 2,856(98.077) & $2,856.36(98.089)$ & 0.0001 & \\
\hline & \multirow{2}{*}{ Total } & \multirow{2}{*}{$2,912(100.00)$} & \multirow{2}{*}{$2,912.00(100.00)$} & 1.9763 & \\
\hline & & & & $P>0.05$ & \\
\hline
\end{tabular}

Note: NA, not available; 14 cases of $\mathrm{S}^{-} \mathrm{S}^{-}$phenotypes were not counted.

\section{DISCUSSION}

Subgroup A2 was the most common subgroup among group A phenotypes except subgroup Al. Subgroup A2 accounts for about $20 \%$ in Caucasians, but is relatively rare in Asians. Subgroups A2 and $\mathrm{A} 2 \mathrm{~B}$ account for $1 \%$ 9\% of groups $\mathrm{A}$ and $\mathrm{AB}$ in the Chinese population ${ }^{[2]}$. In the Xinjiang Kazakhs' population, the detection rate of subgroup $\mathrm{A} 2$ in $\mathrm{A}$ and $\mathrm{AB}$ phenotypes were $7.08 \%$ and $21.79 \%$, respectively. The detection rate of group A was lower than that in Caucasians, while that of group AB was slightly higher than that found in Caucasians. This was also higher than the detection rate of the subgroup $\mathrm{A} 2$ in $\mathrm{A}$ phenotype $(6.45 \%)$ and subgroup $\mathrm{A} 2$ in $\mathrm{AB}$ phenotype $(12.05 \%)$ in the Kazakh population as reported by a blood group investigation ${ }^{[3]}$. It was also significantly higher than that reported by Ying et al. ${ }^{[4]}$, which showed that the subgroup A2 accounted for $0.41 \%$ of group A, while the subgroup A2B accounted for $3.83 \%$ of group $\mathrm{AB}$ in the Chinese population (by tube method). In a study conducted in Shanghai ${ }^{[5]}$, the A2 subgroup accounted for $0.52 \%$ of group A, and the A2B subgroup accounted for $4.03 \%$ of group $A B$, the subgroup A2 accounted for $0.24 \%$ of group $A$ and the subgroup A2B accounted for $5.28 \%$ of group $\mathrm{AB}^{[5]}$. However, the detection rate of subgroup A2 in group $\mathrm{AB}$ was higher than that in group $\mathrm{A}$, which is consistent with other studies ${ }^{[3-6]}$. The data suggested that the frequency of the subgroup A2 in the Kazakh population was between the Caucasian and the Han population. The frequencies of anti-Al antibody found in subgroups A2 and A2B were 1\% 8\% and 22\% 35\%, respectively ${ }^{[7]}$. Due to the combination of the high distribution of subgroup A2 in the Kazakh population and the low blood donation rate, patients with group A or group $A B$ in Kazakh have a higher incidence of producing anti-Al antibodies after transfusion. Thus, more clinical attention should be paid.

$\mathrm{M}$ allele frequency in the MNS blood group system in the Xinjiang Kazak population was 0.566 8, which was higher than that of Wuhu population $(0.4879)^{[8]}$, Shaoguan population $(0.5489)^{[9]}$, Jiangxi Han nationality population $(0.5428)^{[10]}$, Xinjiang Hui people $(0.5204)^{[11]}$, and those detected in northern China $(0.5013)^{[2]}$. It was however, slightly lower than that found in Han people in Chengdu, Sichuan $(0.5752)^{[12]}$, and the Xinjiang Uygur population $(0.5791)^{[13]}$, and lower than that of the Tibet Tibetan population $(0.6809)^{[14]}$ and Jino (0.755 0), Blang (0.670 0) and Lahu (0.715 0) nationalities in Yunan as reported by Xiao et al. ${ }^{[15]}$. It was still lower than that observed in the Xinjiang Yili Kazakh population $(0.7365)^{[16]}$. In this research, the frequency of the $M$ gene in the Xinjiang Kazakh population was consistent with the results reported previously, which found that the $\mathrm{M}$ gene in minority nationality was generally higher than that found in the Han population ${ }^{[17]}$. The distribution of the MN blood group phenotype was characterized as $\mathrm{MN}>\mathrm{MM}>\mathrm{NN}$, which was also in line with previous reports ${ }^{[9-15]}$, with the exception of that found in Wuhu and $\mathrm{Xi}^{\prime}$ an $(\mathrm{MN}>\mathrm{NN}>\mathrm{MM})^{[18]}$. There are few reports on the frequency of the $\mathrm{S}$ and s gene in China. Du et al. found the frequency of the $\mathrm{S}$ and s gene highly differentiated 
in all populations in the world ${ }^{[17]}$, reporting that the frequency of the $\mathrm{S}$ gene was $0.03 \sim 0.04$ in Chinese, but notably higher at $0.3 \sim 0.4$ in Caucasians. The $\mathrm{S}$ gene frequency in the Kazak population in Xinjiang $(0.1860)$ was significantly higher than that of the Han population in Chengdu, Sichuan $(0.0376)^{[12]}$, blood donors in Xi'an $(0.053)^{[18]}$ and the Hui population in Xinjiang $(0.0954)^{[11]}$, was slightly higher than that of Tibetan population $(0.1467)^{[14]}$ and closer to Xinjiang Uygur population $(0.1743)^{[13]}$. In this survey, the phenotypic frequency of SS (3.87\%), Ss (29.18\%) and ss (66.21\%) in the Xinjiang Kazakh population was compared with the SS (8.69\%), Ss $(24.35 \%)$, and ss $(66.96 \%)$ in the Indian population ${ }^{[19]}$, finding that SS phenotypic frequency in Xinjiang Kazakhs was lower than that in Indians, Ss phenotypic frequency was slightly higher than that in Indians, and the ss of the two are more consistent. A total of 1,885 Kazakh individuals were investigated for $\mathrm{S}$ and $\mathrm{s}$ genes in this investigation, 14 cases of rare $\mathrm{S}^{-} \mathrm{s}^{-}$phenotypes were found, accounting for $0.74 \%$, which was lower than that of the Xinjiang Hui population $(2 / 220,0.90 \%)^{[1]]}$ and Xinjiang Uygur population $(4 / 158,2.53 \%)^{[13]}$. This study suggested that the distribution of alleles in Xinjiang Kazakhs with the MNSs blood group system was different from the Han, similar to that of Xinjiang Uygur population, and had its own national characteristics.

The P blood group system showed significant differences among different races and nationalities. The $\mathrm{Pl}$ gene frequency of African people appears higher than that of East Asians, and that of Caucasians resides between the two ${ }^{[17]}$. In this survey, the frequency of the $\mathrm{Pl}$ gene of the Kazak population in Xinjiang $(0.2848)$ was higher than that of Shaoguan population $(0.1574)^{[9]}$, the Han nationality population in Jiangxi $(0.1750)^{[10]}$ and other ethnic minorities in China as published in $2004(0.2140)^{[17]}$. The average frequency of the Pl gene was far lower than that of the Caucasian population $(0.5161)^{[2]}$ and those of Jino (0.435 9), Blang (0.469 0) and Lahu nationalities (0.5099) in Yunnan reported by Xiao et al. ${ }^{[15]}$. It was also slightly lower than that found in the Yili Kazakh population $(0.3252)$ and Uygur population in Xinjiang $(0.3480)^{[16]}$.

The Kell blood group system is the third largest blood group system after $\mathrm{ABO}$ and $\mathrm{Rh}$ in Europe and America ${ }^{[18]}$. The frequency of the $\mathrm{K}$ antigen is lower in all populations, but relatively speaking, is slightly higher in Caucasians, followed by Africans, and lowest in those East Asians ${ }^{[17]}$. This study shows that $\mathrm{K}$ antigen distribution frequency $(1.92 \%)$ and $\mathrm{K}$ gene frequency $(0.0096)$ in the Xinjiang Kazakh population are higher than that of the Xinjiang Hui people (1.36\%, 0.006 8), while lower than the Xinjiang Uygur popu- lation $(3.16 \%, 0.015$ 8) and Caucasians in England $(9.02 \%, 0.0462)$. Unlike the genotyping study of the Kell blood group antigen in 332 blood donors in the Chengdu area by Hong et al. ${ }^{[12]}$, the $\mathrm{K}$ antigen gene frequency $(\mathrm{K}=0.0000, \mathrm{k}=1.0000)$ is also different from that found by Bao et al. ${ }^{[20]}$, in their investigation of the frequency of $\mathrm{K}$ antigen in 10,057 blood donors in Shanghai. In the Chinese Han population, the frequency of $\mathrm{K}$ antigen was $0.06 \%$, and the frequency of $\mathrm{K}$ gene was $0.0017^{[12]}$. Because of the strong immunogenicity of the Kell blood group antigen, both anti$\mathrm{K}$ and anti-k can cause hemolytic transfusion reaction and neonatal hemolytic disease. The distribution of $\mathrm{K}$ gene frequency in the Xinjiang Kazakh population is far higher than that of the Han nationality. Thus it is suggested that in the process of transfusion and prevention of neonatal hemolysis in Kazakh population, attention should be paid to not only the RhD blood group, but also Kell blood group antigens and antibodies. Patients who have developed antibodies should be allocated appropriate blood, autotransfusion, or matching blood from relatives for transfusion after irradiation.

In summary, the phenotype distribution of subgroup A2, blood groups MNS, P, and Kell in the Kazakh population in northern Xinjiang has the characteristics of its own nation, which can be viewed as a kind of infiltration and integration between those commonly found in Western populations and those of East Asian descent. These results provide a large sample of data for anthropology, ethnic group genetics, and add much to the viability of our rare blood group bank for ethnic minorities existing in Xinjiang, and also provide a theoretical basis for formulating transfusion strategies and methods for Kazakh populations.

\section{References}

[1] Xiao XF. Blood transfusion technical manual. Chengdu: Sichuan Science and Technology Press, 1992:68-70.

[2] Zhao TM. Genetics of Human Blood Groups. Beijing: Science Press, 1987: 229-30, 351-5, 364-7.

[3] Blood group investigation team. Investigation on the blood group of 16 nationalities in China I. ABO blood group and $\mathrm{ABH}$ secretion ability investigation. Chin J Hematol (in Chinese), 1980, 1(5): 261-3.

[4] Ying Y, Hong X, Xu X, et al. Serological characteristic and mo-lecular basis of A2 subgroup in the Chinese population. Transfus Apher Sci, 2013, 48(1) : 67-74.

[5] Xiang D, Liu X, Guo ZH, et al. Studies of ABO subgroups in the Chinese Han population in Shanghai area. Chin J Blood Transfusion (in Chinese), 2006, 19(1): 256.

[6] Xie JW, Deng SZ, Lan WL, et al. Genotypic study of ABO subtypes in Panyu area. Chin J Blood Transfusion 
(in Chinese), 2016,29(4): 381-4.

[7] Liu DZ. Immunohematology. Shanghai: Shanghai Scientific \& Technical Publishers, 2002: 39.

[8] Cha Y, Zhao R, Huang H, et al. Investigation of ABO, $\mathrm{Rh}, \mathrm{MN}$ and $\mathrm{P}$ blood groups of the Chinese Han blood donors in Wuhu. Journal of Clinical Transfusion and Laboratory Medicine (in Chinese), 2010, 12( 3): 266-7.

[9] Luo HQ, Yuan X, Liu KB, et al. Distribution of ABO, $\mathrm{Rh}, \mathrm{MN}, \mathrm{P}$ and $\mathrm{Jk}(\mathrm{a}-\mathrm{b}-)$ blood groups in population in Shaoguan prefecture, Guangdong province, China. Chinese Journal of Biologicals (in Chinese), 2008, 21(6): 524-6.

[10] Li GL, Xiao L, Sun Y, et al. Studies on ABO, Rh, MN, Kidd, Lewis and P blood group systems in the Chinese Han population in Jiangxi. Chin J Blood Transfusion (in Chinese), 2001, 14(A1): 98.

[11] Lin GY, Shan JJ, Zhang YN, et al. Distribution of gene frequencies of 9 kinds of rare blood group system in Hui nationalities in Xinjiang, China. Journal of Xinjiang Medical University (in Chinese), 2016,39(8):1026-31.

[12] Hong Y, Gong TX, Zhou CH, et al. Study on the genotyping of nine blood group system antigens by Kell et al. Chin J Blood Transfusion (in Chinese), 2012, 25(8): 763-5.

[13] Lin GY, Du XL, Shan JJ, et al. Molecular genetic analysis of genes from MNS, Duffy and Kell blood groups in the China Xinjiang Uygur population. Chinese Journal of Tissue Engineering Research (in Chinese), 2016, 20(1):
$123-7$.

[14] Zhang R, Tian L, Li XJ, et al. Gene polymorphism of 10 blood group systems in Tibetans, China. Chin J Blood Transfusion (in Chinese), 2014, 27(5): 505-7.

[15] Xiao H, Hao LP, Zhang MQ, et al. Distribution of four red cell blood group system in Jino, Blang and Lahu ethnic groups. Acta Anthropologica Sinica (in Chinese), 2000, 19(3) : 238-43.

[16] Xu YH, Zhang YL, Ai QH, et al. Distribution of ABO, $\mathrm{MN}, \mathrm{P}$ and $\mathrm{Rh}$ blood groups in Xinjiang Uygur, Kazak and Mongolian. Acta Anthropologica Sinica (in Chinese), 1993,12(3):290-3.

[17] Du RF. chinese population genetics. Beijing: Science Press, 2004: 108, 111-3, 129, 139.

[18] Peng J, Liang YL, Xu H, et al. Study on MNS blood group and genetic polymorphism of blood donors in $\mathrm{Xi}$ 'an. Chin J Blood Transfusion (in Chinese), 2017, 30(6): 590-2.

[19] Kahar MA, Patel RD. Phenotype frequencies of blood group systems (Rh, Kell, Kidd, Duffy, MNS, P, Lewis, and Lutheran) in blood donors of south Gujarat, India. Asian J Transfus Sci, 2014, 8(1): 51-5.

[20] Bao YQ, Ding SE, Xie YZ, et al. Investigation of K antigen frequency of 10057 blood donors in Shanghai area. Journal of Clinical Transfusion and Laboratory Medicine (in Chinese), 2003, 5( 3): 203-4.

(Received 11 July 2018, Revised 02 September 2018, Accepted 05 September 2018) 